\title{
Density variations in liquid tellurium: Roles of rings, chains, and cavities
}

\author{
J. Akola, ${ }^{1,2,3}$ R. O. Jones, ${ }^{1,4}$ S. Kohara, ${ }^{5}$ T. Usuki, ${ }^{6}$ and E. Bychkov ${ }^{7}$ \\ ${ }^{1}$ Institut für Festkörperforschung, Forschungszentrum Jülich, D-52425 Jülich, Germany \\ ${ }^{2}$ Nanoscience Center, Department of Physics, University of Jyväskylä, P.O. Box 35, FI-40014 Jyväskylä, Finland \\ ${ }^{3}$ Department of Physics, Tampere University of Technology, P.O. Box 692, FI-33101 Tampere, Finland \\ ${ }^{4}$ German Research School for Simulation Sciences, FZ Jülich and RWTH Aachen University, D-52425 Jülich, Germany \\ ${ }^{5}$ Research \& Utilization Division, JASRI/SPring-8, 1-1-1 Kouto, Sayo-cho, Sayo-gun, Hyogo 679-5198, Japan \\ ${ }^{6}$ Graduate School of Science and Engineering, Yamagata University, 1-4-12 Kojirakawa, Yamagata 990-8560, Japan \\ ${ }^{7}$ Laboratoire de Physico-Chimie de L'Atmosphère, UMR 8101 CNRS, Université du Littoral, F-59140 Dunkerque, France
}

(Received 11 February 2010; published 12 March 2010)

\begin{abstract}
Liquid tellurium has been studied by density-functional/molecular-dynamics simulations at 560, 625, 722, and $970 \mathrm{~K}$ and by high-energy x-ray diffraction (HEXRD) at $763 \mathrm{~K}$ and $973 \mathrm{~K}$. The HEXRD measurements agree very well with earlier neutron-scattering data of Menelle et al. The density maximum near the melting point $(722 \mathrm{~K})$ reflects the competition between twofold and threefold local coordination, which results in chain formation and changed ring statistics at lower $T$, and the variation with $T$ of the volume of cavities (26-35\% of the total). A higher-order gradient expansion of the exchange-correlation functional is needed to describe structural details. Changes in the electronic properties (band gap and dc conductivity) upon cooling are consistent with a transition from a high-temperature metal to a semiconductor.
\end{abstract}

DOI: 10.1103/PhysRevB.81.094202

PACS number(s): 61.20.Ja, 61.05.cp, 71.15.Pd, 71.22.+i

\section{INTRODUCTION}

The remarkable properties of liquid elemental tellurium have challenged our understanding for more than 50 years. At $626 \pm 3 \mathrm{~K}$ there are extrema in the specific heat, thermalexpansion coefficient, compressibility, and related quantities ${ }^{1}$ that point to a structural phase transition, and there is a semiconductor-metal (SC-M) transition close to the melting point $T_{m}(722 \mathrm{~K})$. Most striking is the maximum in the density near $T_{m}$ (Refs. 1 and 2) that is also found in water. ${ }^{3}$ There is continuing controversy about the origin of this very unusual feature in both materials. ${ }^{4}$ Although water and tellurium differ profoundly in their interactions (hydrogen vs covalent bonds), constituents (atoms vs molecules), and structures, the above thermodynamic quantities are astonishingly similar on temperature scales normalized to the respective melting points. ${ }^{3}$ Detailed studies of liquid Te could then provide insight into the critical behavior of water, the most important liquid on Earth, particularly because Te can be supercooled to temperatures on this scale well below that at which water crystallizes spontaneously. However, scattering experiments in Te have led to structure factors with considerable variation, ${ }^{5,6}$ and it has been notoriously difficult to calculate structure factors and pair-correlation functions that resemble those measured. ${ }^{7-11}$ This may be due in part to the relatively small samples considered in previous DF/MD simulations (64, 80, and 125 atoms per unit cell in Refs. 8, 9, and 11, respectively).

Crystalline Te comprises parallel helices (bond length $2.835 \AA$ ) separated by $3.491 \AA{ }^{12}$ and structural models of liquid Te have been proposed with domains of twofold and threefold coordination, both without ${ }^{13}$ and with ${ }^{14-16}$ chains. A reverse Monte Carlo analysis of liquid Te (Ref. 16) indicated the presence of voids and rings. Furthermore, densityfunctional (DF) calculations in Te are unusually sensitive to the choice of exchange-correlation functional, ${ }^{8,17}$ and no sat- isfactory agreement between theory and experiment has been found to date. Here we present high-energy $x$-ray diffraction (HEXRD) data at 763 and $973 \mathrm{~K}$ and the results of DF simulations that agree well with them. The density anomaly and the SC-M transition in liquid Te can be understood in terms of large cavities (voids) and rapid changes in the mediumrange structure. This description does not rely on longerranged density inhomogeneities, such as the metal/ semiconductor domains proposed in Te (Ref. 18) or lowdensity/high-density regions in water. ${ }^{19}$

\section{HIGH-ENERGY X-RAY DIFFRACTION}

The HEXRD experiments were performed at the beamline BL04B2 at SPring-8 (Ref. 20) using a two-axis diffractometer dedicated to glass, liquid, and amorphous materials and a high- $T$ furnace. The incident energy of $\mathrm{x}$ rays was $113.4 \mathrm{keV}\left(Q_{\max }=25 \AA^{-1}\right)$. The sample was sealed in a silica glass tube $(2 \pm 0.05 \mathrm{~mm}$ inner diameter and $0.21 \pm 0.05 \mathrm{~mm}$ wall thickness), and diffraction patterns of samples in this tube and an empty tube were measured in vacuum in a transmission geometry. The collected data were corrected using a standard program, ${ }^{21}$ and the absorption coefficients, ${ }^{22}$ incoherent-scattering functions, ${ }^{23}$ and atomic scattering factors ${ }^{24}$ were used for correction and normalization, leading to the Faber-Ziman total structure factor $S(Q) .{ }^{25}$

The radial distribution function (RDF) or pair-correlation function $g(r)$ is the spherically averaged distribution of interatomic vectors,

$$
g(r)=\frac{1}{\rho^{2}}\left\langle\sum_{i} \sum_{i \neq j} \delta\left(\vec{R}_{i}\right) \delta\left(\vec{R}_{j}-\vec{r}\right)\right\rangle,
$$

where $\vec{R}_{i}$ are the coordinates of atom $i$ and $\rho$ is the number density. In molecular-dynamics (MD) simulations $g(r)$ can be calculated directly from these coordinates, and experi- 

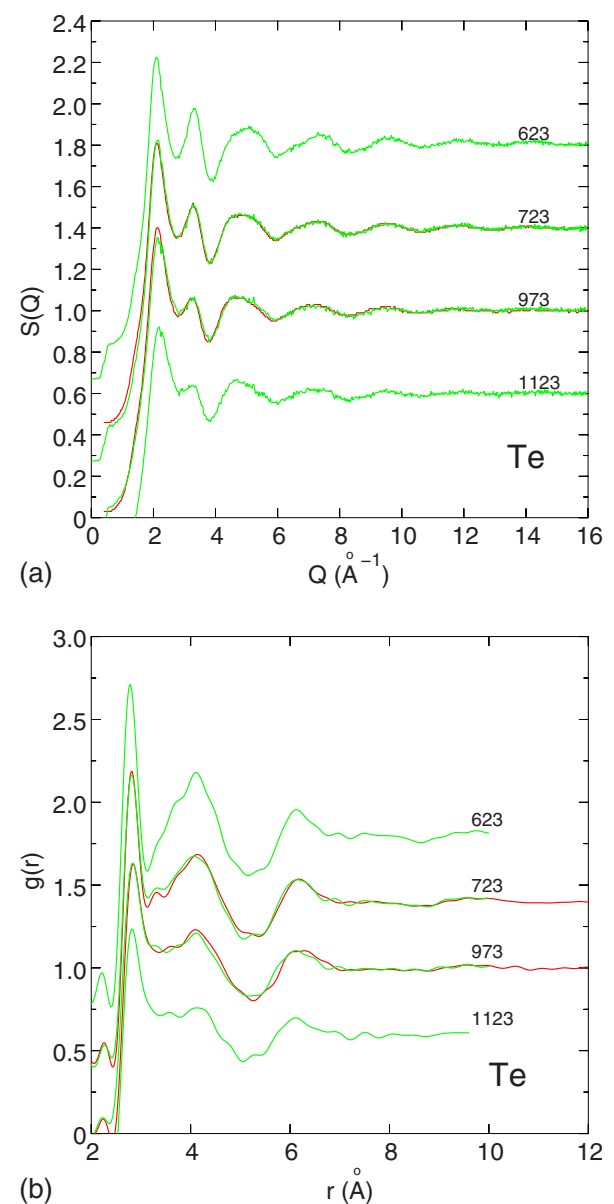

FIG. 1. (Color online) (a) Structure factor $S(Q)$ and (b) radial distribution function $g(r)$ for $\ell$-Te. Red (dark gray): present HEXRD data (763 and $973 \mathrm{~K}$ ); green (light gray): neutrondiffraction data $(623,723,973$, and $1123 \mathrm{~K}$, Ref. 5).

mental values can be determined by Fourier transformation of $S(Q)$,

$$
g(r)=1+\frac{1}{2 \pi^{2} \rho r} \int_{Q_{\min }}^{Q_{\max }} d Q Q[S(Q)-1] \sin (Q r) .
$$

In Fig. 1 we compare our HEXRD measurements of $S(Q)$ and $g(r)$ with neutron-diffraction (ND) results at several temperatures.5 A value of $Q_{\max }$ of $16 \AA^{-1}$ was used in the Fourier transformation in both cases.

\section{DENSITY FUNCTIONAL CALCULATIONS}

The DF/MD calculations used the CPMD program (Born-Oppenheimer mode), ${ }^{26}$ as described elsewhere. ${ }^{27}$ We used time steps of $6.050 \mathrm{fs}$ (initialization) and $3.025 \mathrm{fs}$ (data collection), scalar-relativistic Troullier-Martins pseudopotentials, ${ }^{28}$ and periodic boundary conditions with a single point $(\mathbf{k}=0)$ in the Brillouin zone. The large time steps were made possible by using a stable and efficient predictorcorrector algorithm. ${ }^{29}$ We apply a Nosé-Hoover-chain thermostat $^{30}$ (frequency $800 \mathrm{~cm}^{-1}$, chain length 4), and the rms error in the temperature during the simulations ranged
TABLE I. Structure of crystalline Te (hexagonal cell) for different $E_{x c}$ functionals. $a, c$ : lattice parameters; $r_{0}, r_{\mathrm{d}}$ : intra- and interchain bond lengths; $\alpha$ : intrachain bond angle; $E_{c}$ : cohesive energy; and $E_{\mathrm{d}}$ : interchain component of $E_{c}$.

\begin{tabular}{lccccccc}
\hline \hline & $\begin{array}{c}a \\
(\AA)\end{array}$ & $\begin{array}{c}c \\
(\AA)\end{array}$ & $\begin{array}{c}r_{0} \\
(\AA)\end{array}$ & $\begin{array}{c}r_{\mathrm{d}} \\
(\AA)\end{array}$ & $\begin{array}{c}\alpha \\
(\mathrm{deg} .)\end{array}$ & $\begin{array}{c}E_{c} \\
(\mathrm{eV})\end{array}$ & $\begin{array}{c}E_{\mathrm{d}} \\
(\mathrm{eV})\end{array}$ \\
\hline LDA & 4.29 & 5.87 & 2.90 & 3.30 & 100.4 & 3.28 & 0.48 \\
PBE & 4.51 & 5.89 & 2.90 & 3.48 & 100.8 & 2.42 & 0.19 \\
PBEsol & 5.04 & 5.84 & 2.81 & 3.95 & 102.6 & 3.12 & 0.05 \\
BLYP & 5.11 & 5.91 & 2.84 & 4.01 & 102.8 & 2.00 & 0.04 \\
TPSS & 4.55 & 5.90 & 2.85 & 3.55 & 102.4 & 2.28 & 0.12 \\
Exp. $^{\mathrm{a}}$ & 4.456 & 5.921 & 2.835 & 3.491 & 103.1 & $2.06^{\mathrm{b}}$ & \\
\hline \hline
\end{tabular}

${ }^{\text {aReference } 12 .}$

${ }^{\mathrm{b}}$ Reference 39.

from 26 to $43 \mathrm{~K}$ between 560 and $975 \mathrm{~K}$. The Perdew-BurkeErnzerhof (PBE) (Ref. 31) and TPSS (Ref. 32) approximations were employed for the exchange-correlation energy $E_{x c}$. The kinetic-energy cutoff of the plane-wave basis was 20 Ry (40 Ry for $c$-Te, Table I), and the minimization of the energy functional during the simulations used the direct inversion of the iterative subspace. A detailed analysis of the electronic structure for selected geometries (snapshots) has been carried out with the Lanczos diagonalization scheme and the free-energy functional of Alavi et al. $(T=1000 \mathrm{~K}) .^{33}$ We have used the experimental density for liquid Te (Ref. 1) (Table II) at each $T$.

Table I summarizes the properties of crystalline Te for different $E_{x c}$ functionals. TPSS results are consistently good, while the local-density approximation (LDA) overestimates the cohesive energy and leads to a short interchain distance, and PBE overestimates the bond length $r_{0}$. We also show the

TABLE II. Calculated (TPSS) properties of $\ell$-Te. $r_{0}\left(r_{\min }\right)$ : first maximum (minimum) in RDF; $N$ : total coordination number (cutoff $3.2 \AA$ ); $C_{2}$ : fraction of twofold (including onefold) coordinated Te; $\left\langle\ell\left(C_{2}\right)\right\rangle$ : chain length; $V_{\mathrm{c}}$ : fraction of cavities; $\langle v\rangle$ : average cavity size; $W\left(\varepsilon_{f}\right)$ : DOS at the Fermi level (relative to $970 \mathrm{~K}$ ); $\sigma(0)$ : dc conductivity $(\omega=0) ; P$ : pressure; and $D$ : diffusion constant.

\begin{tabular}{lcccc}
\hline \hline Temperature & $560 \mathrm{~K}$ & $625 \mathrm{~K}$ & $722 \mathrm{~K}$ & $970 \mathrm{~K}$ \\
\hline$\rho\left(\right.$ atoms $\left./ \AA^{3}\right)$ & 0.02618 & 0.02653 & 0.02725 & 0.02653 \\
$r_{0}(\AA)$ & 2.82 & 2.84 & $2.87\left(2.98^{\mathrm{a}}\right)$ & 2.91 \\
$r_{\min }(\AA)$ & 3.28 & 3.60 & 3.71 & 3.77 \\
$N(\mathrm{Te})$ & 2.39 & 2.39 & $2.45\left(2.58^{\mathrm{a}}\right)$ & 2.40 \\
$C_{2}(\%)$ & 66.7 & 63.8 & 59.0 & 60.9 \\
$\left\langle\ell\left(C_{2}\right)\right\rangle$ & 2.80 & 2.48 & 2.15 & 2.07 \\
$V_{\mathrm{c}}(\%)$ & 34.8 & 32.3 & 26.2 & 34.4 \\
$\langle v\rangle\left(\AA^{3}\right)$ & 80.1 & 76.7 & 67.4 & 78.3 \\
$W\left(\varepsilon_{F}\right)$ & 0.57 & 0.75 & 0.81 & 1.00 \\
$\sigma(0)\left(\Omega^{-1} \mathrm{~cm}^{-1}\right)$ & 800 & 1400 & 2400 & 2700 \\
$P\left(\mathrm{kbar}^{3}\right.$ & $1.4 \pm 8.1$ & $3.8 \pm 9.4$ & $-2.3 \pm 9.7$ & $-7.6 \pm 9.9$ \\
$D\left(10^{-5} \mathrm{~cm}^{2} / \mathrm{s}\right)$ & 0.89 & 1.54 & $2.40\left(2.69^{\mathrm{a}}\right)$ & 4.62 \\
\hline \hline
\end{tabular}

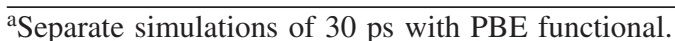


structural parameters found using the PBEsol (Ref. 34) and BLYP approximations. ${ }^{35,36}$ PBEsol has the same form as PBE, but with parameters optimized for extended systems. While the PBEsol description of bond distances (lattice constants) is generally improved for chalcogenide alloys, ${ }^{37,38}$ it overestimates significantly the interchain distance and cohesive energy of $c$-Te. The BLYP form leads to poor results for the lattice constant $a$ and the interchain distance, which arises from a shallow potential-energy minimum, but other results are satisfactory.

The simulation of $\ell$-Te was started at $3000 \mathrm{~K}$ on a $343-$ atom s.c. geometry in a cubic cell (side $23.47 \AA$ ) appropriate to the density at $970 \mathrm{~K}$. After cooling (110 ps, PBE functional) and data collection at $970 \mathrm{~K}(10 \mathrm{ps}$, TPSS $)$, the temperature was lowered to $T_{m}(722 \mathrm{~K}, 86 \mathrm{ps}, \mathrm{PBE})$, where data were collected (18 ps, TPSS). The samples were initialized at each temperature ( $2 \mathrm{ps}$, TPSS) prior to data collection. The procedure was repeated for $625 \mathrm{~K}$ (structural phase transition) and $560 \mathrm{~K}$ (density minimum), where the times for cooling (PBE) and data collection (TPSS) were 60/60 ps and $11 / 10 \mathrm{ps}$, respectively. The total simulation time was $413 \mathrm{ps,}$ and the results are summarized in Table II.

The pressure on the (fixed) cell was small throughout, and its sign was consistent with the density changes. The dependence of the pressure (stress) on the cutoff in the plane-wave basis has been tested by performing calculations with cutoffs of $30 \mathrm{Ry}$ and $40 \mathrm{Ry}$ for three selected snapshots at each temperature. The calculated pressures were within the error bars quoted in Table II in all cases. The diffusion constant $D$ can be calculated from the velocity-velocity autocorrelation function $C_{v}(t)$

$$
D=\frac{1}{3} \int_{0}^{\infty} d t C_{v}(t)
$$

or directly from the coordinates $\vec{R}$

$$
D=\lim _{t \rightarrow \infty} \frac{\left\langle|\vec{R}(t)-\vec{R}(0)|^{2}\right\rangle}{6 t} .
$$

\section{RESULTS}

\section{A. Structure factor, pair distribution function, and coordination numbers}

The structure factors $S(Q)$ measured by HEXRD at 763 and $973 \mathrm{~K}$ agree remarkably well with the ND results of Menelle et al. ${ }^{5}$ [see Fig. 1(a)]. This is an important result since previous scattering measurements on liquid Te have not led to a consistent picture. Apart from minor discrepancies in the peak heights at 2.2 and $3.2 \AA^{-1}$ and the shape of the third maximum at $4.5 \AA^{-1}$, the TPSS calculations show the best agreement with experiment reported to date for DF methods, although the differences in $S(Q)$ increase as $T$ are lowered to $625 \mathrm{~K}$ [see Fig. 2(a)]. This probably reflects the lower atomic mobility that requires even longer simulations. The PBE result is less satisfactory: the second maximum is shifted and broadened, and the phase of the high- $Q$ oscillations differs from experiment.
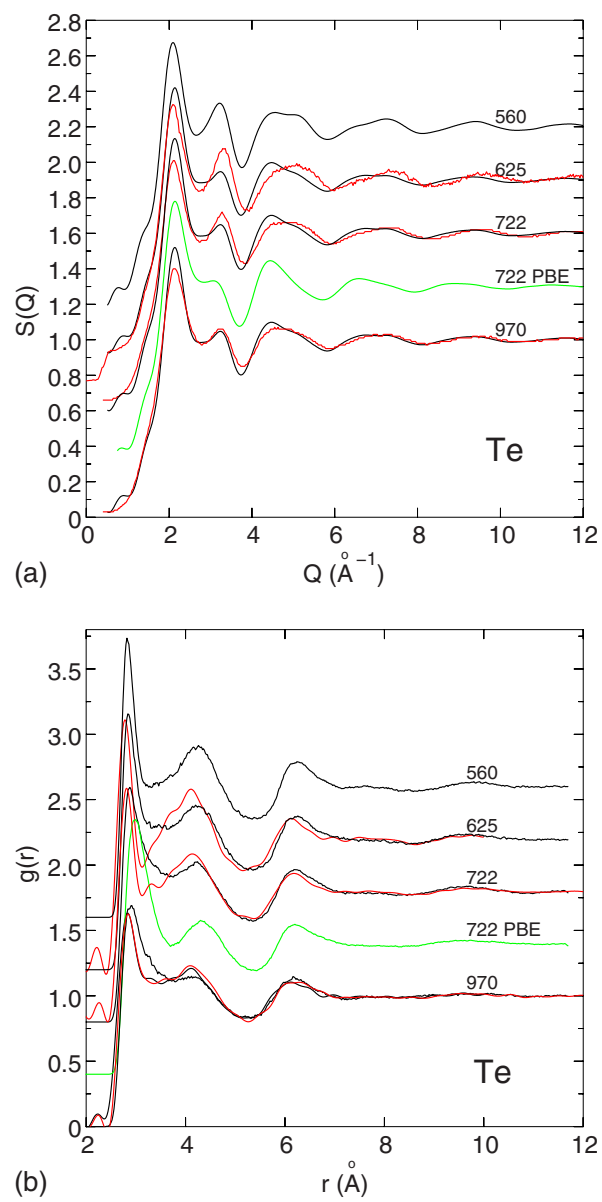

FIG. 2. (Color online) (a) Structure factor $S(Q)$ of $\ell$-Te at 560, 625, 722, and $970 \mathrm{~K}$ (TPSS calculation), with XRD data for 763 and $973 \mathrm{~K}$, and neutron-diffraction data for $623 \mathrm{~K}$ [red (dark gray)]. The PBE result for $722 \mathrm{~K}$ [green (light gray)] is also shown. (b) Corresponding radial distribution functions $g(r)$.

The differences in $S(Q)$ are reflected in the radial distribution functions [RDF, Fig. 2(b)]. The experimental RDF show narrow first peaks at $722(2.80 \AA)$ and $970 \mathrm{~K}$ $(2.83 \AA)$, and a minimum near $3.2 \AA$. The most prominent features (narrow first peak, increased weight in the second peak) are present in the TPSS calculations, but the first peak is broader and the bonds $\sim 2 \%$ longer, and there is more weight at interchain distances 3.2-3.7 $\AA$. The distributions of near-neighbor separations (Fig. 3) show that the first two

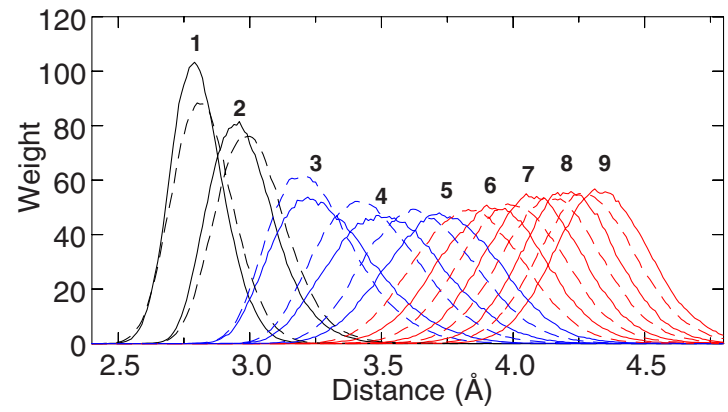

FIG. 3. (Color online) Nearest-neighbor distributions of Te atoms in $\ell$-Te at $560 \mathrm{~K}$ (full curves) and $722 \mathrm{~K}$ (dashed curves). 
TABLE III. Calculated coordination numbers as a function of cutoff $R_{c}$, with HEXRD values (exp) at 763 and $973 \mathrm{~K}$.

\begin{tabular}{ccccccc}
\hline $\begin{array}{c}R_{c} \\
(\AA)\end{array}$ & $560 \mathrm{~K}$ & $625 \mathrm{~K}$ & $722 \mathrm{~K}$ & $763 \mathrm{~K}(\exp )$ & $970 \mathrm{~K}$ & $973 \mathrm{~K}(\exp )$ \\
\hline 3.1 & 2.04 & 2.00 & 1.99 & 2.13 & 1.94 & 1.92 \\
3.2 & 2.39 & 2.39 & 2.45 & 2.49 & 2.40 & 2.34 \\
3.3 & 2.74 & 2.78 & 2.91 & 2.87 & 2.86 & 2.73 \\
3.4 & 3.13 & 3.19 & 3.37 & 3.32 & 3.28 & 3.15 \\
\hline \hline
\end{tabular}

(2.80 and $2.98 \AA$ ), which comprise the first peak of the RDF at $560 \mathrm{~K}$, are significantly shorter than the interchain distances. The short bonds are longer and interchain separations shorter at $722 \mathrm{~K}$ so that the position of the first minimum in $g(r)$ shows a pronounced $T$ dependence as the overlap increases.

Integration over the first peak of $g(r)$ to the experimental minimum $(3.2 \AA)$ yields TPSS coordination numbers $N(\mathrm{Te})$ at 722 and $970 \mathrm{~K}(2.45$ and 2.40) close to the HEXRD results (2.49 and 2.34). The PBE results show longer bonds and overcoordination, a tendency also found in Te clusters. ${ }^{40}$ If we classify the atomic coordination as twofold (or less, $C_{2}$ ) or threefold (or higher, $C_{3}$ ), the former contribute to chains analogous to the helices in the crystal. They form the majority at each $T$ (see the average coordination numbers in Table II), and their fraction is highest $(66.7 \%)$ at $560 \mathrm{~K}$. For completeness we provide in Table III the coordination numbers calculated for cutoffs between 3.1 and $3.4 \AA$. Also
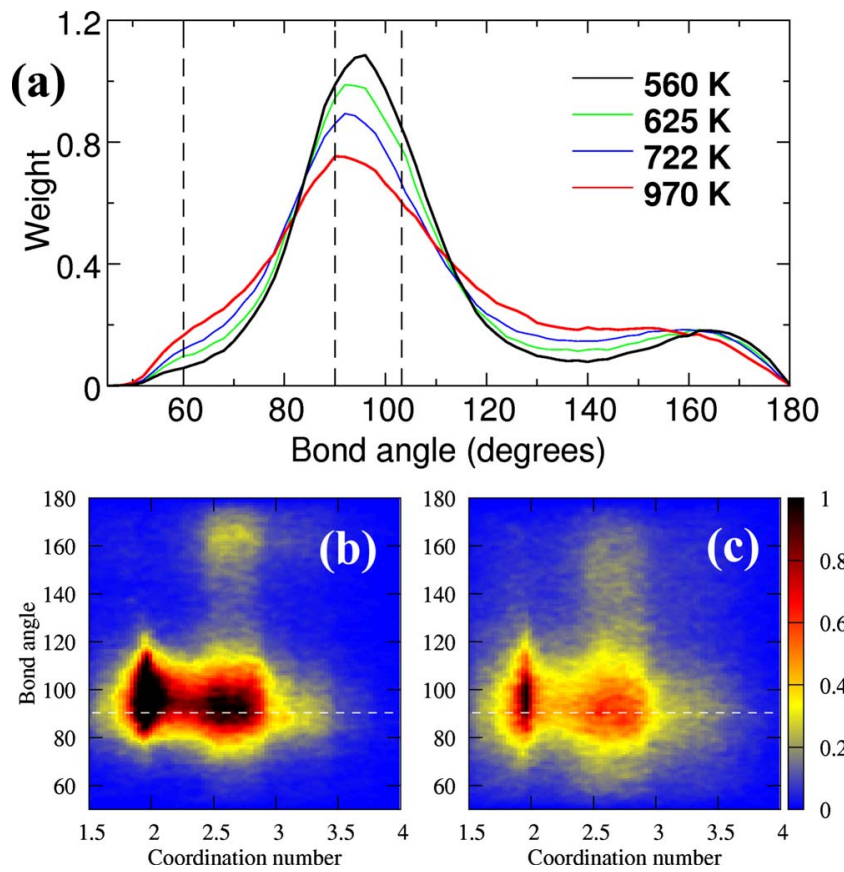

FIG. 4. (Color online) (a) Angular distributions at 560 (sharpest peak), 625, 722, and $970 \mathrm{~K}$. Dashed lines at 60, 90, and $103.2^{\circ}$ are for atomic close packing, octahedral (cubic) coordination, and helices in $c$-Te, respectively. The bond cutoff is $3.2 \AA$. (b) and (c) Angular distributions at 560 and $970 \mathrm{~K}$ as a function of coordination number (smooth cutoff around $3.2 \AA$ ). Dashed white line: $90^{\circ}$.
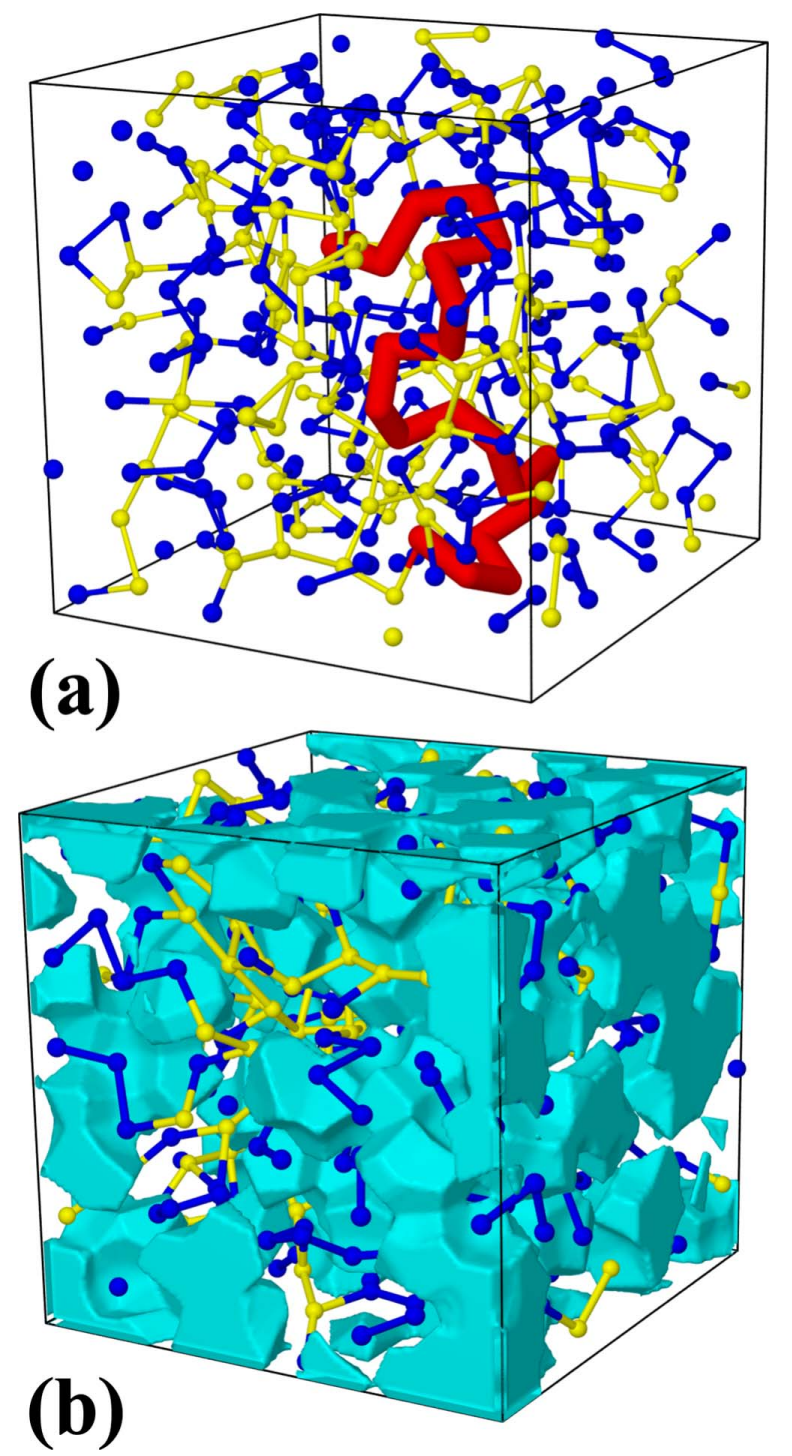

FIG. 5. (Color online) Chains and cavities in snapshot of $\ell$-Te at $560 \mathrm{~K}$ : (a) a 21-membered chain of Te (red); (b) cavities (cyan, $30.1 \%$ of volume). Blue: twofold (or onefold) atoms; yellow: threefold (or higher) coordinated atoms.

shown are the coordination numbers obtained from reverse Monte Carlo analyses of the HEXRD data at $763 \mathrm{~K}$ and $973 \mathrm{~K}$

\section{B. Bond angle distributions}

The bond angle distributions [Fig. 4(a)] show octahedral features, with a strong peak at $90^{\circ}$ and a weaker peak at $180^{\circ}$. Both broaden gradually as $T$ increases and provide few signs of the density anomaly. The maximum shifts from 96 to $90^{\circ}$ upon heating from 560 to $970 \mathrm{~K}$, and triangular configurations (bond angles near $60^{\circ}$ ) are more evident. Figures 4(b) and 4(c) show that twofold atoms have strongly peaked distributions near $100^{\circ}$ and few linear configurations, while Te atoms with higher coordination show octahedral bond angles. The distribution at $970 \mathrm{~K}$ is significantly broader, but the narrow range of twofold Te atoms remains evident. We 


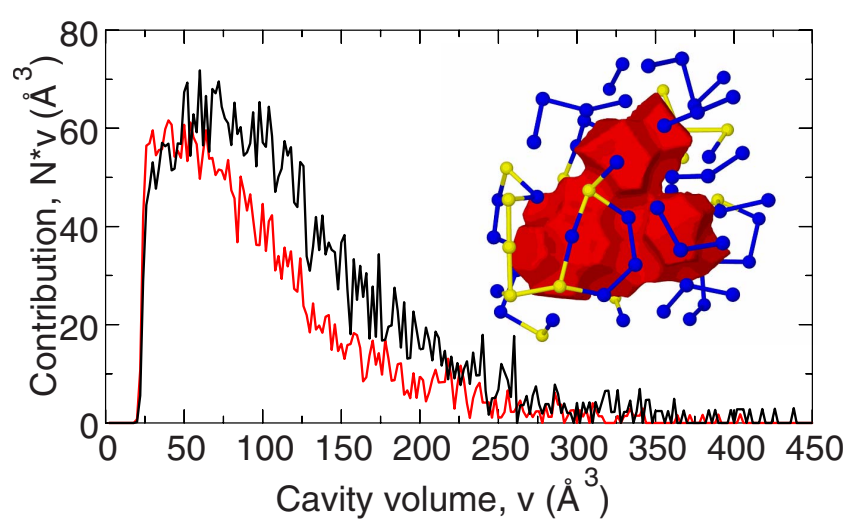

FIG. 6. (Color online) Cavity volumes at $560 \mathrm{~K}$ (black, upper curve) and $722 \mathrm{~K}$ (red) weighted by their contribution in total volume. Inset: large cavity and environment (333 $\left.\AA^{3}, 560 \mathrm{~K}\right)$.

find no evidence of short-long alternation in the intrachain bonds, and they do not prefer linear configurations. ${ }^{7,11}$

\section{Chains, rings, and cavities}

Information about the density anomaly point requires simulation cells large enough to describe chains and cavities [Figs. 5(a) and 5(b)], i.e., beyond the coordination numbers, bond lengths, and bond angles accessible to small samples. Chains are characteristic of liquid Te and can be terminated either by a threefold Te (not included in the chain) or a onefold Te (included) and can have over 20 links at $560 \mathrm{~K}$ [Fig. 5(a)]. The bond angles in such chains are similar to those in $c$-Te [Figs. 4(b) and 4(c)], but the interchain distances vary widely and allow numerous empty regions: "cavities" (voids, vacancies).

Cavities have been analyzed by determining points that are farther from any atom than a given cutoff (here $2.8 \AA$ ) and building cells around them according to the Voronoi prescription. ${ }^{27}$ A similar method has been applied recently to study cavities in amorphous and liquid $\mathrm{Sb}_{2} \mathrm{Te}_{3} .{ }^{41}$ Cavities in Te have complicated shapes, often far from spherical, ${ }^{16}$ and occupy between $26 \%$ and $35 \%$ of the total volume (Table II), depending on $T$. Their volume contributions as a function of size are shown in Fig. 6 for at $560 \mathrm{~K}$ (density minimum) and $722 \mathrm{~K}$ (density maximum). Cavities with up to 10 atomic volumes $\left(\sim 300 \AA^{3}\right)$ are not uncommon. Larger cavities (50-250 $\AA^{3}$ ) are more abundant at $560 \mathrm{~K}$, where the total cavity volume is $8.6 \%$ larger than at $722 \mathrm{~K}$. Since this increase is greater than the density change $(4.1 \%)$, the volume ascribed to atoms increases at the maximum density $(722 \mathrm{~K})$.

The distributions of irreducible rings, i.e. closed paths along bonds, differ dramatically at 560 and $722 \mathrm{~K}$ [Fig. 7(a)]. Larger rings dominate at $560 \mathrm{~K}$, small and intermediate rings $(<20)$ at $722 \mathrm{~K}$. Most twofold coordinated atoms occur in very short chains [Fig. 7(b)], but the chains are longer at 560 $\mathrm{K}$. The average coordination numbers (560 K: 2.39; $722 \mathrm{~K}$ : 2.45) are very similar, however, so that the short-range structures must differ significantly. The increased tendency to chain formation at lower $T$ is reflected in fewer small rings, and threefold coordinated atoms cluster and form branching sites between the entangled chains/rings [Fig. 7(c)]. These
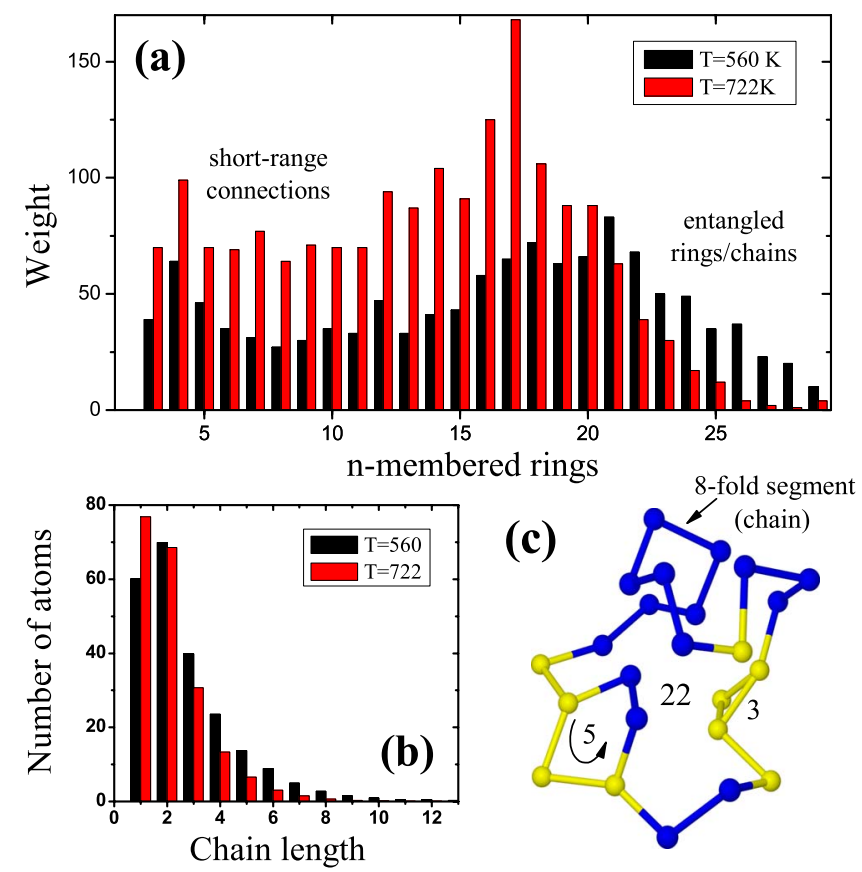

FIG. 7. (Color online) (a) Statistics of irreducible $n$-membered rings at 560 and $722 \mathrm{~K}$. (b) Size distribution of chains. (c) A large, partially entangled, ring of 22 members with two smaller rings, and chains (blue atoms). Yellow: atoms with higher coordination.

changes are closely related to the cavity distribution, as many rings surround cavities. There are more small rings and fewer large cavities at $722 \mathrm{~K}$.

Ring statistics and the cavity analysis provide information about the probability that two atoms in a network are connected by bonds. Large cavities-especially those with irregular shapes-reduce such connections, and more highly coordinated atoms increase them by acting as branching sites. Classical simulations using the TIP5P force field ${ }^{42}$ indicate that cavity volumes, ring sizes, and changes in the local coordination are also crucial to understand the anomalous density variations of water at low $T$. This work is being extended and will be described elsewhere. Such analyses go beyond the short-range atomic arrangement, and several order parameters have been used (e.g., orientational and translational order). ${ }^{43}$

\section{Diffusion}

The diffusion constant $D$ has been evaluated directly from the motion of the atoms (Eq. (4)) and is given for four temperatures in Table II. Measurements of the self-diffusion coefficient in Te have been made using neutron scattering, ${ }^{44}$ where the broadening of the elastic peak caused by diffusion of the atoms in a liquid can be related to $D,{ }^{45}$ and by observing the motion of radioactive tracer atoms ( a capillary geometry. ${ }^{46}$

The experimental data are characterized by substantial error bars and difficulties in extrapolating values measured at higher temperatures to the melting point. Nevertheless, the agreement between experiment and calculated values is reasonable: quasielastic neutron scattering gives a value of $D$ of 
$2.6 \pm 0.6 \times 10^{-5} \mathrm{~cm}^{2} / \mathrm{s}$ at $723 \mathrm{~K}$ (our value is 2.40 ), capillary data extrapolated to $T_{m}$ give $D=1.8$ in the same units.

\section{E. Electronic properties}

The Kohn-Sham eigenvalues $\psi_{i}$ and eigenfunctions $\varepsilon_{i}$ differ from the many-particle wave functions of states of the system, and calculations of electronic excitations using them often lead to discrepancies with measured spectra. It is well known, for example, that many approximations for $E_{x c}$ lead to underestimates of the band gap in semiconductors and insulators. Nevertheless, such calculations often lead to qualitative insight into the electronic properties and are used here in this spirit. In Te, a gap in the electron density of states (DOS) emerges gradually on cooling, and this is monitored in Table II as the ratio of the DOS at the Fermi energy $W\left(\varepsilon_{F}\right)$ to its value for the metallic system at $970 \mathrm{~K}$. A similar $T$ dependence of the DOS was found by Zhao et al. ${ }^{11}$

The eigenvalues $\varepsilon_{i}$ and eigenfunctions $\psi_{i}$ can also be used to estimate the conductivity using the Kubo-Greenwood expression $^{47}$

$$
\sigma(\omega)=\frac{2 \pi e^{2}}{3 m^{2} V} \frac{1}{\omega} \sum_{i, j}\left(f_{i}-f_{j}\right)\left|\left\langle\psi_{i}|\vec{p}| \psi_{j}\right\rangle\right|^{2} \delta\left(\varepsilon_{i}-\varepsilon_{j}-\hbar \omega\right),
$$

where the $f_{i}$ denote occupation numbers, and $V$ the cell volume. The finite size of the unit cell means that the eigenvalue spectrum is discrete, and the dc conductivity is found by polynomial extrapolation of $\omega \rightarrow 0$. The decrease in this estimate of the dc conductivity from 2700 (at $970 \mathrm{~K}$ ) to $800 \Omega^{-1} \mathrm{~cm}^{-1}$ (at $560 \mathrm{~K}$ ) (Table II) is consistent with the increasing band gap.

\section{CONCLUDING REMARKS}

DF/MD simulations (343 atoms, over $400 \mathrm{ps)} \mathrm{on} \mathrm{liquid} \mathrm{Te}$ at $560,625,722$, and $970 \mathrm{~K}$ show that the TPSS functional ${ }^{32}$ describes Te better than other approximations in widespread use. The TPSS approximation was designed to give good results for molecules and solids, and our positive experience with a molecular solid is consistent with the original expectations. ${ }^{32,48}$ New HEXRD measurements at $763 \mathrm{~K}$ and $973 \mathrm{~K}$ agree well with previous ND data. ${ }^{5}$ This is an important result, because earlier scattering data on liquid Te had not led to a consistent picture.

The rapid changes in the atomistic structure that occur in liquid Te can be understood without invoking the existence of domains. Twofold and threefold coordinated atoms are present, and the density anomaly arises from temperaturedependent changes in chain lengths, ring distributions, and cavity volumes. The supercooled liquid at $560 \mathrm{~K}$ resembles $c$-Te, but the absence of translational order leads to cavities in the interchain regions and the density minimum. Preliminary calculations indicate that cavities are also essential to understand the density variations in water. In Te, DF simulations on samples with several hundred atoms are needed to understand these features, since the short-range structure (coordination number, bond lengths and angles) does not suffice. The SC-M transition, evident in changes in both the band gap and the dc conductivity, is related to the broadening of bond angle range at higher $T$ also found in amorphous $\mathrm{Ge}_{2} \mathrm{Sb}_{2} \mathrm{Te}_{5}{ }^{49}$

\section{ACKNOWLEDGMENTS}

The HEXRD experiment was approved by JASRI (Proposal No. 2009A1207), and the calculations were performed on IBM Blue Gene/P and p6 575 computers in the FZ Jülich with grants from the FZJ and the John von Neumann Institute for Computing (NIC). We thank C. Bichara for discussions and providing the original data of Ref. 5, and the Academy of Finland (J.A.), the Japan Science and Technology Agency (S.K. and T.U.), and the European Union Interreg IVA Program (E.B.) for support.
${ }^{1}$ Y. Tsuchiya, J. Phys.: Condens. Matter 3, 3163 (1991).

${ }^{2}$ H. Thurn and J. Ruska, J. Non-Cryst. Solids 22, 331 (1976).

${ }^{3}$ H. Kanno, H. Yokohama, and Y. Yoshimura, J. Phys. Chem. B 105, 2019 (2001); See also A. Angell, Nat. Nanotechnol. 2, 396 (2007).

${ }^{4}$ The situation in water has been surveyed by T. Tokushima, Y. Harada, O. Takahashi, Y. Senba, H. Ohashi, L. G. M. Pettersson, A. Nilsson, and S. Shin, Chem. Phys. Lett. 460, 387 (2008).

${ }^{5}$ A. Menelle, R. Bellissent, and A. M. Flank, Europhys. Lett. 4, 705 (1987).

${ }^{6}$ H. Endo, K. Maruyama, H. Hoshino, and H. Ikemoto, Z. Phys. Chem. 217, 863 (2003), compare several ND pair-correlation functions.

${ }^{7}$ C. Bichara, J.-Y. Raty, and J.-P. Gaspard, Phys. Rev. B 53, 206 (1996).

${ }^{8}$ R. Stadler and M. J. Gillan, J. Phys.: Condens. Matter 12, 6053 (2000).

${ }^{9}$ K. Seifert-Lorenz, G. Kresse, and J. Hafner, J. Non-Cryst. Solids
293-295, 193 (2001).

${ }^{10}$ D. Molina and E. Lomba, Phys. Rev. B 67, 094208 (2003).

${ }^{11}$ G. Zhao, C. S. Liu, and Z. G. Zhu, J. Phys.: Condens. Matter 20, 335102 (2008).

${ }^{12}$ C. Adenis, V. Langer, and O. Lindqvist, Acta Crystallogr., Sect. C: Cryst. Struct. Commun. 45, 941 (1989).

${ }^{13}$ B. Cabane and J. Friedel, J. Phys. (Paris) 32, 73 (1971); J. R. Magaña and J. S. Lannin, Phys. Rev. Lett. 51, 2398 (1983).

${ }^{14}$ J. Hafner, J. Phys.: Condens. Matter 2, 1271 (1990).

${ }^{15}$ M. Misawa, J. Phys.: Condens. Matter 4, 9491 (1992).

${ }^{16}$ K. Maruyama, H. Endo, and H. Hoshino, J. Phys. Soc. Jpn. 77, 034603 (2008), and references therein.

${ }^{17}$ G. Kresse, J. Fürthmüller, and J. Hafner, Phys. Rev. B 50, 13181 (1994).

${ }^{18}$ Y. Tsuchiya and E. F. W. Seymour, J. Phys. C 18, 4721 (1985).

${ }^{19}$ M. Matsumoto, Phys. Rev. Lett. 103, 017801 (2009).

${ }^{20}$ M. Isshiki, Y. Ohishi, S. Goto, K. Takeshita, and T. Ishikawa, Nucl. Instrum. Methods Phys. Res. A 467-468, 663 (2001). 
${ }^{21}$ S. Kohara, M. Itou, K. Suzuya, Y. Inamura, Y. Sakurai, Y. Ohishi, and M. Takata, J. Phys.: Condens. Matter 19, 506101 (2007).

${ }^{22}$ S. Sasaki, National Laboratory for High Energy Physics, Japan, KEK Report No. 90-16, 1991 see http://ccdb4fs.kek.jp/cgi-bin/ img/allpdf?199024016

${ }^{23}$ J. H. Hubbell, Wm. J. Veigele, E. A. Briggs, R. T. Brown, D. T. Cromer, and R. J. Howerton, J. Phys. Chem. Ref. Data 4, 471 (1975).

${ }^{24}$ D. Waasmaier and A. Kirfel, Acta Crystallogr., Sect. A: Found. Crystallogr. 51, 416 (1995).

${ }^{25}$ T. E. Faber and J. M. Ziman, Philos. Mag. 11, 153 (1965).

${ }^{26}$ CPMD V3.13 Copyright IBM Corp 1990-2009, Copyright MPI für Festkörperforschung Stuttgart 1997-2001 (http:// www.cpmd.org).

${ }^{27}$ J. Akola and R. O. Jones, Phys. Rev. B 76, 235201 (2007); J. Phys.: Condens. Matter 20, 465103 (2008) $\left[\mathrm{Ge}_{2} \mathrm{Sb}_{2} \mathrm{Te}_{5}\right]$; Phys. Rev. Lett. 100, 205502 (2008) [GeTe].

${ }^{28}$ N. Troullier and J. L. Martins, Phys. Rev. B 43, 1993 (1991), the valence configuration is $5 s^{2} 5 p^{4}$.

${ }^{29}$ J. Kolafa, J. Comput. Chem. 25, 335 (2004).

${ }^{30}$ G. J. Martyna, M. L. Klein, and M. Tuckerman, J. Chem. Phys. 97, 2635 (1992).

${ }^{31}$ J. P. Perdew, K. Burke, and M. Ernzerhof, Phys. Rev. Lett. 77, 3865 (1996).

${ }^{32}$ J. Tao, J. P. Perdew, V. N. Staroverov, and G. E. Scuseria, Phys. Rev. Lett. 91, 146401 (2003).

${ }^{33}$ A. Alavi, J. Kohanoff, M. Parrinello, and D. Frenkel, Phys. Rev. Lett. 73, 2599 (1994).

${ }^{34}$ J. P. Perdew, A. Ruzsinszky, G. I. Csonka, O. A. Vydrov, G. E.
Scuseria, L. A. Constantin, X. Zhou, and K. Burke, Phys. Rev. Lett. 100, 136406 (2008).

${ }^{35}$ A. D. Becke, Phys. Rev. A 38, 3098 (1988).

${ }^{36}$ C. Lee, W. Yang, and R. G. Parr, Phys. Rev. B 37, 785 (1988).

${ }^{37}$ J. Akola and R. O. Jones, Phys. Rev. B 79, 134118 (2009).

${ }^{38}$ J. Akola and R. O. Jones, Appl. Phys. Lett. 94, 251905 (2009).

${ }^{39}$ National Bureau of Standards Circular 500, E $E_{\mathrm{c}}$ from Selected Values of Chemical Thermodynamic Properties, (USGPO, Washington, D.C,, 1952), p. 49.

${ }^{40} \mathrm{~J}$. Akola and R. O. Jones (unpublished).

${ }^{41}$ S. Caravati, M. Bernasconi, and M. Parrinello, Phys. Rev. B 81, 014201 (2010).

${ }^{42}$ M. W. Mahoney and W. L. Jorgensen, J. Chem. Phys. 112, 8910 (2000).

${ }^{43}$ See, for example, J. R. Errington and P. G. Debenedetti, Nature (London) 409, 318 (2001).

${ }^{44}$ A. Axmann, W. Gissler, A. Kolmar, and T. Springer, Discuss. Faraday Soc. 50, 74 (1970).

${ }^{45}$ K. S. Singwi and A. Sjölander, Phys. Rev. 119, 863 (1960).

${ }^{46}$ D. H. Kurlat, C. Potard, and P. Hicter, Phys. Chem. Liq. 4, 183 (1974).

${ }^{47}$ R. Kubo, J. Phys. Soc. Jpn. 12, 570 (1957); D. A. Greenwood, Proc. Phys. Soc. London 71, 585 (1958).

${ }^{48}$ V. N. Staroverov, G. E. Scuseria, J. Tao, and J. P. Perdew, Phys. Rev. B 69, 075102 (2004).

${ }^{49}$ J. Akola, R. O. Jones, S. Kohara, S. Kimura, K. Kobayashi, M. Takata, T. Matsunaga, R. Kojima, and N. Yamada, Phys. Rev. B 80, 020201(R) (2009). 